\title{
COVID-19: plasma de convaleciente en la sala de Clínica Médica del Hospital Gral. de Agudos "Dr. Cosme Argerich" en Buenos Aires
}

\author{
Recibido: 11/11/20 Aceptado: 20/11/20
}

Marcelo Zylberman ${ }^{1}$, José L. Unrein ${ }^{2}$, Leandro Heffner ${ }^{1}$, Clarisa Gashu', Agostina Bruno ${ }^{1}$, Jorge Rodríguez ${ }^{3}$, Juan Gagliardi', Esteban Cejas² ${ }^{2}$ Cecilia Blanco ${ }^{2}$, Nora Lescano², Diego Vega², Liliana Calderone ${ }^{2}$, Miriam Monelos², Marcelo Arias², Silvia Pilar², Viviana Ramundo², Mario Romano², Adrián Guevara², Ana Gancitano², César Sánchez².

\section{RESUMEN}

Se presentan los resultados de seguridad y evolución clínica de 87 pacientes que recibieron plasma de convaleciente en la sala de Clínica Médica del Hospital Argerich en Buenos Aires. Tres pacientes tuvieron sobrecarga de volumen. Hubo 33 pases a Terapia Intensiva $(37,9 \%)$ y 21 casos requirieron asistencia respiratoria mecánica $(24,1 \%)$. Fallecieron 18 pacientes $(20,7 \%)$. Tres de ellos por limitación del esfuerzo terapéutico y 15 en Terapia Intensiva. La mortalidad en Terapia Intensiva fue del $45,4 \%$. Hubo solo 10 casos que recibieron plasma dentro de las 72 horas del comienzo de síntomas: de ellos, 1 caso de asistencia respiratoria y 1 fallecido; entre 72 horas y una semana recibieron plasma 25 casos con $26,9 \%$ de asistencia respiratoria y $20 \%$ de mortalidad, y de los 50 pacientes que recibieron mas allá de la primera semana, $25,4 \%$ requirieron de asistencia respiratoria y $24 \%$ fallecieron. Nuestra serie no pudo demostrar efecto beneficioso en la administración temprana de plasma y no fue diseñada para medir eficacia. El procedimiento fue bien tolerado en la gran mayoría de los pacientes.
1 División Clínica Médica, Hospital Gral. de Agudos “Dr. Cosme Argerich" GCBA. Buenos Aires, Argentina. ${ }^{2}$ Medicina Transfusional, Hospital Gral. de Agudos "Dr. Cosme Argerich" GCBA. Buenos Aires, Argentina.

${ }^{3}$ Terapia Intensiva, Hospital Gral. de Agudos “Dr. Cosme Argerich" GCBA. Buenos Aires, Argentina.

${ }^{4}$ Comité de Etica e Investigación, Hospital Gral. de Agudos "Dr. Cosme Argerich" GCBA. Buenos Aires, Argentina.

Dirección para la correspondencia: Prof. Dr. Marcelo Zylberman. Olaya 1341. CP 1414. CABA. Cel: 1144379117.

Email:mzylberman@fibertel.com.ar

Estudio sin financiación. Los contenidos del material no han sido publicados anteriormente. Quienes figuran como autores participaron significativamente en la investigación o elaboración del manuscrito y se hacen responsables de todo su contenido. Ninguno de los autores presenta conflicto de intereses en relación a esta publicación.

Palabras clave: plasma, convaleciente, COVID-19, sala. 


\section{Introducción}

En marzo 2020 se conoció que cuatro pacientes en China habían mejorado de su situación crítica de SARSCoV-2 con transfusión de plasma de convaleciente (1); se sumaron diez pacientes con buenos resultados publicados en PNAS (2) y, en abril, una revisión sistemática publicada en Medical Virology concluyó que era un procedimiento seguro y beneficioso (3).

Los 5000 casos (4) y luego 20.000 casos de la Mayo Clinic (5) abrieron la posibilidad que la transfusión de plasma de convaleciente en forma temprana se transformara en una herramienta útil para el manejo de los pacientes con COVID-19.

Por ello, en el Hospital Argerich se diseñó un protocolo de acceso extendido para el uso de plasma con la participación de los servicios de Clínica Médica, Medicina Transfusional y Terapia Intensiva.

Con los objetivos de:

- conocer el requerimiento de pase a Terapia Intensiva (UTI), el requerimiento de asistencia respiratoria mecánica (ARM) y la mortalidad entre los pacientes a quienes se les administra plasma de convaleciente SARS-CoV-2 en la sala de Clínica Médica;

- conocer si hay relación entre la fecha de inicio de síntomas/administración de plasma con la evolución clínica y

- evaluar la seguridad de la administración de plasma de convaleciente en pacientes con neumonía COVID-19

se revisaron los datos de los pacientes transfundidos en Clínica Médica y que fueron incluidos en el estudio prospectivo de acceso expandido de Plasma de Convaleciente del Hospital Argerich, del Gobierno de la Ciudad De Buenos Aires.

\section{Materiales y métodos}

Se incluyeron, en forma consecutiva, pacientes ingresados por neumonía por COVID-19.

Los criterios de inclusión fueron:

- $\quad$ Edad igual o mayor a 18 años.
- Paciente con diagnóstico confirmado de infección respiratoria por COVID-19 por RT-PCR.

- Diagnóstico por radiografía, tomografía o ecografía de tórax de imagen de lesión pulmonar consistente con neumonía y $\mathrm{SaO}_{2} \leq 93 \%$ con cánula nasal a 4 litros y/o frecuencia respiratoria mayor a 30 por minuto.

- Hospitalización por la infección respiratoria en sala general.

- El sujeto o el familiar o el allegado en condiciones de prestar el consentimiento informado.

Fueron criterios de exclusión:

Imposibilidad o negativa para consentir por parte del familiar o su representante legal.

- Paciente embarazada (por determinación de BHCG en edad fértil).

- Contraindicación para recibir infusión de plasma por historia de anafilaxia a productos sanguíneos o por alto riesgo de sobrecarga de volumen asociada a la transfusión.

- Paciente con criterios de shock refractario definido por dosis de noradrenalina mayor a $1 \mathrm{ug} / \mathrm{k} / \mathrm{min}$ por más de dos horas consecutivas.

- Coagulopatía severa por la que se requiera administración de plasma fresco congelado.

- Pacientes en hemodiálisis o bajo ECMO.

Deterioro neurológico secundario a proceso hemorrágico cerebral.

- Signos de isquemia miocárdica activa.

Para la selección de donantes de plasma se exigieron las siguientes condiciones:

1. Resolución de síntomas igual o más de 14 días previos a la donación.

2. Hombres o mujeres (no embarazadas con confirmación de BHCG si en edad fértil). 
3. Edad comprendida entre 18-65 años.

4. Resultados negativos en dos pruebas de PCR en hisopado nasofaríngeo o una PCR negativa a los 21 días del inicio de los síntomas.

5. Ser no reactivo para las serologías habituales de los donantes de sangre: Chagas (HAI/QMIA), HBV antígeno de superficie y anticuerpo anticore, HCV antígeno y anticuerpo, HIV, HTLV1, sífilis y brucelosis.

6. Tener una hemoglobina superior a $11 \mathrm{~g} / \mathrm{dl}$.

7. Firma del consentimiento informado específico.

Todos los pacientes recibieron la transfusión de una unidad de PC (200 a $250 \mathrm{ml}$ ) previo estudio de compatibilidad. El plasma fue obtenido por aféresis manual en todos los casos y solo se infundieron aquellos con títulos de lgG de 1/400 o mayor por metodología ELISA (COVID Ar, Lab. Fundación Leloir). Se registraron los datos demográficos, comorbilidades conocidas, saturación medida por oximetría de pulso y datos de laboratorio como glóbulos blancos, linfocitos, ferritina y proteína $\mathrm{C}$ reactiva (PCR) así como el tiempo de inicio de los síntomas (FIS) hasta la infusión de plasma.

El modo de egreso se registró como: alta, pase a UTI (entre ellos, aquellos que requirieron ARM) y óbito.

Se consideraron como puntos finales de seguridad primarios:

- Deterioro rápido del estado respiratorio o clínico durante la transfusión de plasma convaleciente de SARS-CoV-2.

- Incidencia acumulada de eventos adversos graves durante el período de estudio: reacción a la transfusión (fiebre, erupción cutánea), lesión pulmonar aguda relacionada con la transfusión (TRALI), sobrecarga circulatoria asociada a la transfusión (TACO), o infección relacionada con la transfusión.

\section{Análisis estadístico}

Las variables cualitativas se presentan como valores absolutos y su porcentaje y las variables cuantitativas como media \pm desvío estándar (DE) o mediana e intervalo intercuartilo (IIC) según el análisis de normalidad realizado mediante el test de Shapiro-Wilk. Para el análisis de las variables discretas se utilizó el test de chi cuadrado (con corrección de Yates) o prueba exacta de Fisher. Para el análisis de los datos continuos se utilizó el test de t o test no paramétrico (Wilcoxon y Kruskall Wallis) según su distribución.

Se utilizó para el análisis el programa Epilnfo versión 7.2.4 y Stata/SE® 13.0 para Windows. Se consideró significativo un valor $p<0.05$

\section{Aspectos éticos}

Todos los pacientes dieron su consentimiento para su participación en el estudio.

El protocolo fue aprobado por el Comité de Ética en Investigación del Htal. Gral. Agudos Dr. Cosme Argerich y registrado en el Registro de Protocolos de Investigación del Ministerio de Salud del Gobierno de la Ciudad de Buenos Aires (PRIISABA) con el número 1821.

\section{Resultados}

Entre el 19/6 y el 10/9 de 2020 se incluyeron 87 pacientes con diagnóstico confirmado de infección respiratoria por COVID-19 por RT-PCR internados en la sala de Clínica Médica del Hospital Argerich.

De ellos, 52 (59,8\%) eran hombres y 35 mujeres $(40,2 \%)$. La edad promedio fue de 57,4 $\pm 13,9$ años (rango 21 a 88 años) sin diferencias de acuerdo al género.

Todos los pacientes se hallaban bajo tratamiento con dexametasona al ingresar al protocolo y ninguno recibió

Tabla 1. Datos demográficos y clínicos al ingreso

\begin{tabular}{l|l}
\hline Cantidad de pacientes & $\mathrm{n}: 87$ \\
\hline Hombres & $\mathrm{n}: 52(59,8 \%)$ \\
\hline Mujeres & $\mathrm{n}: 35(40,2 \%)$ \\
\hline Edad en años & $57,4(21-88)$ \\
\hline $\begin{array}{l}\text { Saturación de } 02 \text { al aire } \\
\text { Saturación de O2 con máscara } \\
\text { reservorio }\end{array}$ & $88,6 \pm 2,4 \%$ \\
\hline Glóbulos blancos & $96,6 \pm 1,5 \%$ \\
\hline Linfocitos & $7900 / \mathrm{mm}^{3}(5800-9400)$ \\
\hline Ferritina & $965 / \mathrm{mm}^{3}(690-1320)$ \\
\hline
\end{tabular}


otro tipo de terapia inmunospresora.

Al ingreso, la saturación de oxígeno al $21 \%$ de $88,6 \pm 2,4 \%$, mientras que la saturación con reservorio fue $96,6 \pm 1,5 \%$.

La mediana de glóbulos blancos al ingreso fue 7900/ $\mathrm{mm}^{3}$ (5800-9400) con 965,5 linfocitos/ $\mathrm{mm}^{3}$ (690-1320). Los valores de ferritina al ingreso fueron $1157 \mathrm{ng} / \mathrm{ml}$ (5372000) (Tabla 1).

La infusión del plasma se realizó con una mediana de 8 (6-10) días desde la FIS. Dos pacientes lo recibieron el mismo día que ingresaron y dos pacientes con más de 20 días de evolución (23 y 51 días).

El $96,5 \%$ de los pacientes $(n=84)$ toleró la infusión sin complicaciones. Solo tres $(3,4 \%)$ presentaron TACO y requirieron pase a UTI.

Se registraron 33 pases a UTI $(37,9 \%)$ y $21(24,1 \%)$ requirieron ARM.

Los egresos a domicilio fueron 67 (77\%). De ellos, 51 fueron desde la sala $(58,6 \%)$ y 16 de pacientes que habían requerido UTI ( $48 \%$ de los pases a UTI). Dos casos permanecen en UTI.

La mortalidad global en este grupo de pacientes fue $20,7 \%$ $(n=18)$. De ellos, tres fueron en sala por limitación del esfuerzo terapéutico (LET) y 15 fueron entre los 21 que recibieron ARM $(71,4 \%)$. La mortalidad global de los 33 pacientes en UTI fue de $45,4 \%$ (Tabla 2).

Diez pacientes recibieron el plasma dentro de las 72 horas de la FIS y falleció 1 de ellos (10\%); 25 pacientes lo recibieron entre las 72 horas y la semana de FIS con un $20 \%$ de mortalidad ( 5 casos), y entre los 50 pacientes que lo recibieron luego de la primera semana la mortalidad fue del $24 \%$ (12 casos). Dos casos permanecen en UTI a la fecha.

Respecto del requerimiento de ARM, 1 de los 10 casos

\section{Tabla 2. Evolución clínica}

\begin{tabular}{l|l}
\hline Cantidad de pacientes & n: 87 \\
\hline Pase a UTI & n: $33(37,9 \%)$ \\
\hline ARM & n: $21(24,1 \%)$ \\
\hline Mortalidad & n: $18(20,7 \%)$ \\
- Mortalidad en UTI & n: $15(45,4 \%$ de los 33 ingresados a UTI) \\
- Mortalidad por LET & n: 3 \\
\hline Egresos a domicilio & n: $67(77 \%)$ \\
- Egresos desde sala & n: $51(58,6 \%)$ \\
- Al regreso de UTI & n: $16(48 \%$ de los 33 ingresados a UTI)
\end{tabular}

antes de las 72 horas, (10\%), 7 de 26 (26,9\%) entre 72 horas y una semana, y 13 de 51 después de la primera semana $(25,4 \%)$.

No se observaron diferencias en la mortalidad por el nivel de glóbulos blancos $(p=0,91)$, linfocitos $(p=0,41)$, ferritina $(p=0,57)$ y PCR $(p=0,29)$.

Tampoco se observó relación en los días desde el inicio de los síntomas y la infusión del plasma con respecto a la mortalidad $(p=0,88)$.

\section{Discusión}

A pesar del entusiasmo inicial con los resultados del plasma de convaleciente en el tratamiento de pacientes con COVID-19, los autores fueron cautos al no contar con estudios randomizados que permitieran medir su eficacia. Mayo Clinic alertó sobre el uso temprano del plasma y le adjudicó el beneficio probable del descenso de la mortalidad entre los primeros 5000 y los subsiguientes 15.000 casos transfundidos (5). El grupo de Houston (6), en un extenso estudio de casos y controles, demostró un descenso en las complicaciones y mortalidad entre aquellos pacientes transfundidos dentro de las 72 horas de comienzo de los síntomas con títulos de anticuerpos iguales o mayores a 1:1350.

Nuestra serie intentó demostrar si el procedimiento era seguro y cómo era la evolución clínica de los pacientes transfundidos. Respecto de la seguridad, el 3,5\% tuvo

\section{Tabla 3. FIS, ARM y mortalidad}

\begin{tabular}{l|c|c|c|}
\hline \multicolumn{1}{|c|}{ Tiempo entre FIS y plasma } & Cantidad de pacientes $\mathbf{n}(\%)$ & ARM $\mathbf{n}(\%)$ & Mortalidad $\mathbf{n}(\%)$ \\
\hline$<72 \mathrm{hs}$ & $10(11,5 \%)$ & $1(10 \%)$ & $1(10 \%)$ \\
\hline 72hs - 1 semana & $26(29,9 \%)$ & $7(26,9 \%)$ & $5(20 \%)$ \\
\hline$->1$ semana & $51(58,6 \%)$ & $13(25,4 \%)$ & $12(24 \%)$
\end{tabular}

2 pacientes permanecen en terapia intensiva 
TACO que requirió pase a UTI. Las complicaciones cardíacas en la serie de Mayo Clinic fue del $3 \%$, similar a la observada en nuestros casos (5). Respecto de la evolución clínica, en el periodo marzo-septiembre la División Clínica Médica del Hospital Argerich admitió 873 internaciones por COVID-19, y de ellos requirieron pase a UTI el 10,5\%, o sea 92 pacientes (Sánchez y col., datos no publicados). De los 87 casos transfudidos con plasma, el 37,9\% fue derivado a UTI, lo que se explica porque los pacientes transfundidos con plasma se hallaban dentro de los que desaturaban bajo oxígeno a 4 litros por minuto a través de cánula nasal y requerían máscaras con reservorio. En 90 controles de la sala de Clínica Médica del Hospital Argerich matcheados por edad y comorbilidades con los pacientes que recibieron plasma, 27 pasaron a UTI (30\%), aunque estos incluyeron casos de pacientes que solo requerían oxigeno por cánula nasal, sin hallarse diferencias estadisticamente significativas entre los casos y controles (Sánchez y col, datos no publicados).

Nuestra serie no pudo demostrar que la administración temprana, sugerida por revisiones sistemáticas (7), impacte en el pase a UTI, requerimiento de ARM o mortalidad, pero el número de pacientes que recibió el plasma antes de las 72 horas de la FIS fue escaso.

Dos estudios randomizados no hallaron beneficio del uso de plasma de convaleciente. EI CON-COVID fue realizado en Holanda y se detuvo precozmente antes de terminar el enrolamiento pues el análisis interino demostró que un alto número de receptores ya tenían anticuerpos neutralizantes antes de recibir el plasma (8).

El PLACID, estudio randomizado indio, observó una mortalidad a 28 días del $13,6 \%$ en el grupo que recibió plasma y $14,6 \%$ en los que recibieron placebo (9).

Estos datos llevaron a un editorial del British Medical Journal con serias advertencias sobre el uso de plasma, su potencial efecto protrombótico y su mínimo efecto beneficioso (10).

En el estudio de la provincia de Buenos Aires, la mortalidad de los pacientes que fueron ingresados a piso y transfudidos fue de $14 \%$, menor a la nuestra, pero allí la indicación del plasma era a solicitud del médico tratante y no por criterio de desaturación (11). Este grupo tampoco demostró beneficios en mortalidad entre transfusión temprana y tardía. En el estudio de CEMIC, la mortalidad a 90 días de los pacientes transfundidos fue de $25,2 \%$, similar a nuestra serie (12). En resumen, nuestra serie no pudo demostrar efecto beneficioso en la administración temprana de plasma y no mide eficacia pues no fue diseñada para ello. El procedimiento fue bien tolerado en la gran mayoría de los pacientes. 


\section{Bibliografía}

1. Zhang B, Liu S, Tan T, Huang W, Dong $Y$, Chen L. Treatment With Convalescent Plasma for Critically III Patients With Severe Acute Respiratory Syndrome Coronavirus 2 Infection. Chest 2020; 158:e9-e13.

2. Duan K, Liu B, Li C, Zhang H, Yu T, Qu J, et al. Effectiveness of convalescent plasma therapy in severe COVID-19 patients. Proc Natl Acad Sci U S A. 2020 Apr 28;117:9490-96.

3. Rajendran K, Krishnasamy N, Rangarajan J, Rathinam J, Natarajan M, Ramachandran A. Convalescent plasma transfusion for the treatment of COVID-19: Systematic review. J Med Virol. 2020;10.1002/ jmv.25961. doi:10.1002/jmv.25961

4. Joyner MJ, et al. Early Safety Indicators of COVID-19 Convalescent Plasma in 5,000 Patients. J Clin Invest. 2020: https://doi.org/ 10.1172/JCI140200

5. Joyner, M. J., Bruno, K. A., Klassen, S. A., Kunze, K. L., Johnson, P. W., Lesser, et al. Safety Update: COVID-19 Convalescent Plasma in 20,000 Hospitalized Patients. Mayo Clinic Proceed 2020; 95: 1888-97.

6. Salazar E, Christensen PA, Graviss EA, Nguyen DT, Castillo B, Chen J, et al. Treatment of COVID-19 Patients with Convalescent Plasma Reveals a Signal of Significantly Decreased Mortality, The American Journal of Pathology (2020), https://doi. org/10.1016/j.ajpath.2020.08.001.

7. Bakhtawar N, Usman $M$, Khan $M$,) Convalescent Plasma Therapy and Its Effects On COVID-19 Patient Outcomes: A Systematic Review of Current Literature. Cureus 2020;12: e9535. DOI 10.7759/cureus.9535

8. Gharbharan A, Jordans C, GeurtsvanKessel C, den Hollander J, Karim F,Mollema F,et al. Convalescent Plasma for COVID-19. A randomized clinical trial. medRxiv 2020.07.01.20139857; https://doi. org/10.1101/2020.07.01.20139857

9. Agarwal A, Mukherjee A, Kumar G, Chatterjee $P_{1}$ BhatnagarT,MalhotraP.thePLACIDTrialCollaborators. Convalescent plasma in the management of moderate COVID-19 in India: An open-label parallel-arm phase II multicentre randomized controlled trial (PLACID Trial). BMJ 2020;m3939.

10. Pathak E. Convalescent plasma is ineffective for covid-19.Lessons from the Placid Trial. BMJ 2020;371:m4072

11. Gonzalez S, Regairaz L, Ferrando N, Gonzalez Martínez V, Salazar M, Estenssoro E. Terapia con plasma de convalecientes en pacientes COVID-19 en la Provincia de Buenos Aires. Medicina (Buenos
Aires) 2020; 80

12. Valentini $R$, Fernandez $J$, Riveros $D$, Pálizas $F$, Solimano J, Saúl P, et al. Plasma de convalecientes como terapia para la neumonía por COVID-19 grave. Medicina (Buenos Aires) 2020; 80 
Convalescent plasma in Internal Medicine Ward from Hospital Argerich in Buenos Aires

We present here the results of safety and clinical outcome of 87 patients that received convalescent plasma transfusion in the internal medicine ward of Hospital Argerich in Buenos Aires. Three patients developed transfusion-associated circulatory overload. Thirty-three patients were admitted to Intensive Care Unit (37,9\%) and 21 cases required mechanical ventilation. Eighteen patients died $(20,7 \%), 3$ of them due to limitation of therapeutic effort and 15 in ICU. The mortality in ICU was $45,4 \%$. Ten patients received plasma within $72 \mathrm{hs}$ from the onset of symptoms. Of them, 1 case required mechanical ventilation and died. Twenty-five patients received plasma between 4 and 7 days from onset of symptoms, with a mortality rate of $20 \%$ and $26,9 \%$ of mechanical ventilation. Fifty patients received plasma past the 7 days from onset of symptoms, with a mortality rate of $24 \%$ and $25,4 \%$ of mechanical ventilation. Our series could not prove positive effects in the early administration of plasma and it was not designed to measure effectiveness. The procedure was well tolerated by most of the patients.

Keywords: plasma, convalescent, COVID-19, ward. 